\title{
Grey system Research and Application in the Pool Game
}

\author{
Tatsuyuki Nezu \\ Department of Chemical and Materials Engineering National Chin-Yi University of Technology \\ No.57,Sec.2, Zhong shanRd., Taiping Dist.,Taichung 41170, Taiwan, ROC \\ E-mail: kelvinyu@ncut.edu.tw
}

\begin{abstract}
Keywords:Grey Theory, Pool Game, Collision Theory
Abstract. The objective of this study is to develop a defensive strategy of the intelligent billiard robot by using the grey system theory. The main purpose of this defensive strategy is to make a "safety play", which is the defensive positioning of the balls to minimize the opponent's chance to score or to make a foul stroke. In accessible balls type, it will be easier for us to win the ball game when opponents have to face a safety play. The experimental results show that the application makes the billiard robot a safety play, which makes it easier to win the billiard game.
\end{abstract}

\section{Introduction}

1.Base on the 9th ball billiard rules, the cue ball can't touch the other non-target balls before collidingThe object ball.

2. There is another non-target ball between the specific route for the target ball and the goal bag of opponent, and the cue ball can't directly target the target ball which can be hit into specific bag.

3. Through this experiment, the best hitting angles $\left(\theta_{t}\right)$ for the opponent are greater than $70^{\circ}$ or less than $-70^{\circ}$, and the opponent can't usually hit the target ball into the goal bag when hitting under these conditions.

\section{Basic Theories}

\section{Grey System Theory}

The "Grey system theory" was established by Pro. Deng Ju-Long(Deng J,1980), Automatic Control and Computer Science Department, Huazhong University of Science and Technology in 1982, in order to research the system pattern which the conditions and information are not clear and incomplete, through the establish system modeling (Relationship Analysis) and the correlation analysis (Model Constructing), and to analyze and understand the system by prediction (Prediction) and decision (Decision). The "Grey theory" is developed by the combination of information and mathematical methods, and it has been widely used in the fields of economic, scientific, applied engineering and ecological assessment(Wu Hanxiong Deng Julong, Wenkun Li,1998).

The pattern of "Grey theory" is established by a small amount of data, whose characteristics are as follows:

(1)It needs 4 sample data at least to establish the "Grey dynamic model".

(2)It needs only 3 samples at minimum in each sequence to establish the" Grey relational analysis model".

(3) The "Grey situation decision" needs 3 samples in each goal.

\section{Grey Situation Decision-Making System}

The "Grey decision" refers to the combination of events, countermeasures and effect (Wukun Li, Zhao Zhongxian, 1998). The adopted countermeasures solve the problem and effect in an event, and the basic assignment for decision-making is selected the best solution to deal with an incident event in a variety of countermeasures and to deal with the same event. The "Grey Decision" is defined as follows:
(1) $M\left(u_{h}^{p}\right)=r_{l h}^{p} \in r^{p} \Rightarrow r_{h h}^{p} \in[0,1](2) r_{l h}^{p} \in U^{+}$

Effect Measure mapping $M$ (transformation) 
a. measure of a ceiling effect to deal with a great goal $M^{p h}=\frac{u_{l h}^{p}}{\max _{h}}\left\{u_{l h}^{p}\right\}$

b. handle moderate target $M$ measure for moderate effect $r_{l h}^{p}=\frac{\min \left\{u_{l h}^{p}, u_{0}\right\}}{\max \left\{u_{l h}^{p}, u_{0}\right\}}$

, which ${ }^{u_{0}}$ is purposed target.

c. lower limit effect measure to deal with a relatively small target $r_{l h}^{p}=\frac{\min _{h}\left\{u_{l h}^{p}\right\}}{u_{l h}^{p}}$

4. For the situation $S_{l t}$ if there is the target $f, " \mathrm{p}=1,2, \ldots$, $f$, under the mapping $M$ is the $M\left(u_{l h}^{p}\right)=r_{l h}^{p}$, is $r_{l h}^{1}, r_{l h}^{2}, \ldots, r_{l h}^{p}$, so the $r_{l h}^{p}$ total value of the some effect measure which is called $r_{l h}^{\Sigma}$ as integrated value. Such as: $r_{l h}^{\Sigma}=\frac{1}{f} \sum_{p=1}^{f} r_{l h}^{p}$

\section{Method}

In this study, the main use of the cue ball is that it can collide with the target ball by the various impact angle and strength, and then the predicted position of the cue ball and object ball after impact can be calculate from the geometric relationship between the balls on the pool. In the "grey decision" system, the best impact strength and angle provide the billiard robot addition to scoring attack, and also improve their own judgment issued by the defensive ability to hit the ball for the further the chance increase of winning sparring with people.

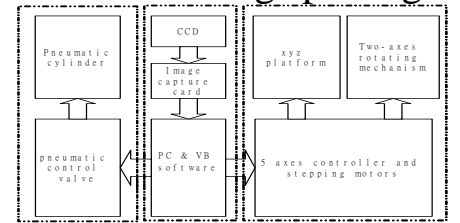

Figure 3.1 Flow chart of the experimental setup

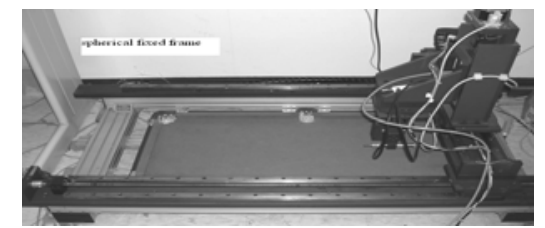

Figure 3.2 Billiard robot

\section{With “Accessibility Balls” type of defense decision-making thinking}

Grey defensive decisions determine blow way through the program impact route, and for billiard robot of batting control. The grey theory is verified by the decision-making of the best batting way, and whether able to hit the cue ball and object ball into the opponent's adverse scoring position or not. Step 1: Visual system two goals distance connection angle is obtained via image, and striking the target ball range $5.20^{\circ}-8.46^{\circ}$ is projected to be the cue ball (Figure 3.4), which divided into seven kinds of angles route (Figure 3.5(1) - (7)), Step 2: Based on the projection coordinates of the cue ball and object ball, the grey pockets select system is taken advantages [16]. The right to decide the best goal of each program bag reanalysis predicts two goals in each program position of their indicator of the sample values.

Step 3: Determine the event, countermeasures and the situation in the target sample.

(1) Event: batting point of view, the choice of force $a_{1}$

(2) Countermeasures: case $1\left(b_{1}\right)$, case $2\left(b_{2}\right) \ldots . .$. case $98\left(b_{b_{9}}\right)$.

(3) Situation

$$
\begin{aligned}
& S_{1,1}=\left(a_{1}, b_{1}\right)=(\text { Launch angle, force, select option } 1) \\
& S_{1,2}=\left(a_{1}, b_{2}\right)=(\text { Launch angle, force, select option } 2) \\
& S_{1,98}=\left(a_{1}, b_{98}\right)=(\text { Launch angle, force, select option } 98)
\end{aligned}
$$

(4) Target: samples with polarity

(i) Target 1: golf bag from the larger-value target (target ball off center, corresponding pockets center distance). When the target ball farther pockets center, the cue ball must pass a larger energy to the target the ball crashed into the bag, scoring relatively difficult to improve, and so defensive measure 
value approaching 1 . The sample unit pixel and its pocket from the sample values, the effect measure

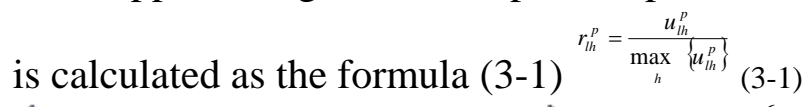

$$
\left\{\begin{array}{lrll}
\text { if } & u_{l h}^{p} \geq 90 & \text { then } & 1 \\
\text { if } & 90 \geq u_{l h}^{p} \geq 60 & \text { then } & \frac{u_{l h}^{p}}{30} \\
\text { if } & 60 \geq u_{l h}^{p} \geq-60 & \text { then } & 0 \\
\text { if } & -90 \geq u_{l h}^{p} \geq-60 & \text { then } & \frac{-u_{l h}^{p}}{30} \\
\text { if } & u_{l h}^{p} \leq-90 & \text { then } & 1
\end{array}\right\}
$$

$$
\left\{\begin{array}{rrrrr}
\text { if } & u_{l h}^{p} \geq 450 \text { then } & 1 \\
\text { if } & 400 \geq u_{l h}^{p} \geq 350 \text { then } & \frac{u_{l h}^{p}-200}{50} \\
\text { if } & 350 \geq u_{l h}^{p} \geq 70 \text { then } & 0 \\
\text { if } & 70 \geq u_{l h}^{p} \geq 30 \text { then } & \frac{u_{l h}^{p}-50}{50} \\
\text { if } & u_{l h}^{p} & \geq 50 \text { then } & 1
\end{array}\right\}
$$

(ii) Target 2: hitting angle (cue ball forward direction and the angle between the directions of the target ball forward): purposed value target sample units for the degree. Batting angle is defined as the connection with the target ball and the cue ball to the point of impact corresponding to the angle between the pockets center connection (Figure 3.6). When the hitting angle is larger, the cue ball striking the target ball will angle variability big, but not conducive to control energy transfer is also smaller and easy to make the opponent cannot score. Therefore, the larger launch angle as possible for the safety ball (Figure 3.7).

(iii) Target 3: cue ball and the point of impact $(G)$ distance purposed value target. When the point of impact with the cue ball is close when the object ball can hit the cue ball and become smaller, target ball forward direction after the impact will be limited, not easy ball crashed into; conversely, when very far away from the cue ball hit the object ball angle change impacts will increase, easily lead to mistakes [19] to the experimental test. When the cue ball and the point of impact within a distance of about $10 \mathrm{~cm}$ or more than $45 \mathrm{~cm}$ (70 pixel and 350 pixel or more), less susceptible to the ball crashed into the pockets. In this study, in order to predict the best goal of the cue ball and object ball, bags may the virtual impact point $(G)$ of the distance relationships sample values; relatively low probability when the distance of the cue ball and the point of impact in this range, the goal. Solid measure value purposed effect measure is shown in Figure 3.8 (sample pixel). And the effect measure value of the cue ball and the point of impact are predicted as a decision-making factor, so effect measure can be defined as shown in Figure 3.3.

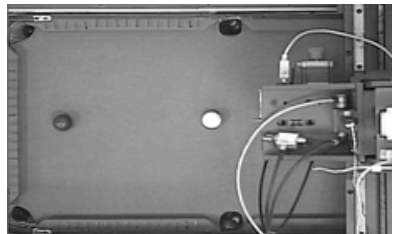

Figure 3.3 Diagram of sphere type impact state accessibility

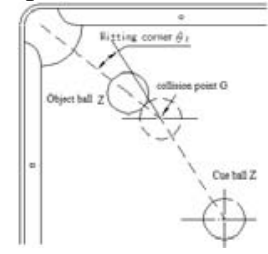

Figure 3.6 Diagram of launch angle

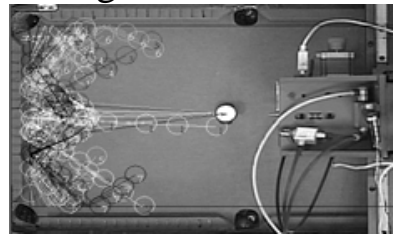

Figure 3.4 Prediction map of sphere type impact accessibility

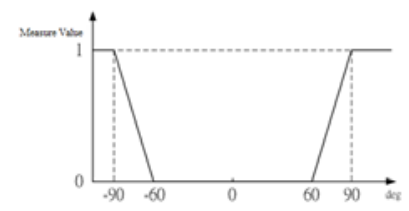

Figure 3.7 Diagram of launch angle effect measure

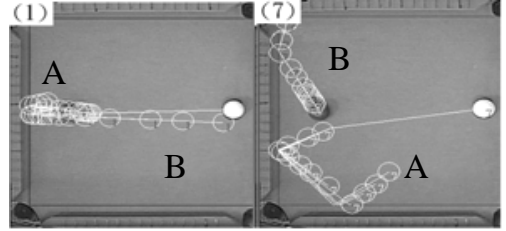

Figure 3.5 Accessibility of the various launch angle of the golf ball-type

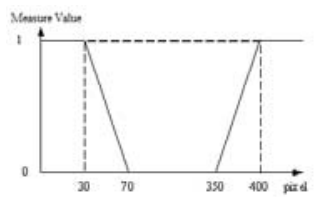

Figure 3.8 Effect of center distance on measuring from cue ball to the target ball

Step 4: the effect measure of sample for processing

Target (i): (specify ball off center and the corresponding pockets distance from the center), with great value target (look big effect measure), impact angle, the force of choice for the event ${ }^{a_{1}}, \mathrm{l}=1$, different programs from 1 to 98, into the formula (3-1) ceiling effect measure formula

$$
r_{l h}^{p}=\frac{u_{1 h}^{1}}{\max _{h}\left\{u_{1 h}^{1}\right\}}=\frac{u_{1 h}^{1}}{581.54}
$$

$$
\text { i.e. } h=1 \quad r_{11}^{1}=\frac{u_{11}^{1}}{581.54}=\frac{389.34}{581.54}=0.669
$$


Target (ii): (launch angle size) purposed value of the target (purposed effect measure), from Figure 3.8 formula (3-2), such as the program launch angle sample $u_{11}^{2}=-15.6^{\circ}$ value sits effect measure value $r_{11}^{2}=0$.

Target (iii): (cue ball and the distance of the point of impact), purposed value target (purposed Effect Measure) program this value $u_{11}^{3}=256.85$ into the formula (3 -3) calculate the effect of obtaining the measure values $r_{11}^{3}=0$

Step 5: the various programs of the decision-making

(i) Find the measure of the combined effect: Considering and experimenting goal difficulty through the general experience of amateur boxers and the three goals, the goal of two (batting angle) is obtained for the impact on the defense strategy. When accessibility ball, the size of the angle will be subjective impact opponent judgment against goals, its weight value is set to 0.6; while considering the goal of a (target ball of center and the corresponding pockets center distance) and target 3 (cue ball and the point of impact distance) as goal difficulty lower target two, the weight value is set to 0.2. Therefore, program is a combined effect of measure:

$r_{11}^{\Sigma}=0.2 \times r_{11}^{1}+0.6 \times r_{11}^{2}+0.2 \times r_{11}^{3} \quad(3-5) \quad=0.2 \times 0.67+0.6 \times 0+0.2 \times 0=0.134$

Take into account a satisfactory decision-making condition, maximum value is as follows:

$$
\begin{aligned}
h^{*}=\arg & \max _{h} r_{l h}^{\Sigma}=\arg \max \left(r_{1,1}^{\Sigma}, r_{1,2}^{\Sigma}, r_{1,3}^{\Sigma}, \ldots \ldots, r_{1,98}^{\Sigma}\right) \\
& =\arg \max (0.134,0.153, \ldots \ldots, 0.169) \\
& =r_{1,8}^{\Sigma}
\end{aligned}
$$

By column $\mathrm{H}$ shows that when $\mathrm{h}=8$; that $S_{l h}=\left(a_{1}, b_{h}\right)=\left(a_{1}, b_{8}\right)$ means that 8 angle impact force, the most difficult opponent in the target ball balls into pockets, and impact angle and force when the grey decision system, which run program controlled the billiard robot to the impact location shot.

\section{Results and Discussion}

In this paper, a defensive strategy of the intelligent billiard robot is developed by using the grey system theory. The optimal stroke is obtained by the angle crashed in to, leading the increase self-determination of billiard robot. The defense and attack ability of billiard robot is supplied, thus the possibility of win can be increased. The main purpose of this defensive strategy is to make a "safety play", which is the defensive positioning of the balls to minimize the opponent's chance to score or to make a foul stroke. The application makes the billiard robot a safety play, which makes it easier to win the billiard game. By the way of modifying for softwares, the sub-soft wares to equip the abilities of robot to meet the change environment along with the editing. The effective improvement of the gaps to the environment is made by using of modified parameters. According to the results of softwares analysis and experiment, the way to make the opponent won't easily to get the goal exactly is verified under the condition of 2 target balls and 1 cue ball by means of "Grey Defense Control".

\section{References}

[1] T. Jebara, C. Eyster, "Augmenting the Billiards Experience with Probabilistic Vision and Wearable Computers,” IEEE Wearable Computers, Vol.13 pp.138-145, Oct 1997.

[2] S. C. Chua, E. K. Wong, Alan W. C. Tan, and V. C. Koo , "Decision Algorithm for Pool Using Fuzzy System," Proceedings of the International Conference on Artificial Intelligence in Engineering \& Technology(ICAIET 2002), , Kota Kinabalu, Malaysia, pp. 370-375, 17-18 June 2002.

[3] A. B. Siegmann and S. Kenig, ”Residual Stress in Polymers III:The Injection-Molding Processes conditions ”Polymer Engineering and Science,Vol.16,pp.560-568, 1976 
[4] S. C. Chua, E. K. Wong and V. C. Koo, "Pool Balls Identification and Calibration for a Pool Robot," Proceedings of the International Conference on Robotics, Vision, Information and Signal Processing (ROVISP 2003), Penang, Malaysia, pp 312-315, 22-24 January 2003. [5] H. Nakama, I. Takaesu and H. Tokashiki, "Basic Study on Development of Shooting Mechanism for Billiard Robot”, JSME, Robotic Workshop.

[6] Deng J. Grey System Theory. Huazhong University of Science \& Technology Press, 1990.

[7] B. R. Cheng and J. S. Yang, 2004, “Design of The Neural-Fuzzy Compensator for a Billiard Robot,” IEEE International Conference on Networking, Sensing and Control (ICNSC'04), Taipei Taiwan, 2004 March, 21-23.

[8] J. S. Yang, Z. M. Lin and C. Y. Yang, “Grey Decision-Making for a Billiard Robot,” IEEE International Conference on Systems, Man \& Cybernetics (SMC’04), Hague, Netherlands, 2004 Oct, $10-13$.

[9] H. Nakama, I. Takaesu and H. Tokashiki, "Basic Study on Development of Shooting Mechanism for Billiard Robot,” JSME, Robotic Workshop, 2001.

[10]Fei Long, Johan Herland, Marie-Christine Tessier, Darryl Naulls, Andrew Roth, Gerhard Roth, Michael Greenspan, “Robotic Pool: An Experiment in Automatic Potting,” IROS’04, Sendai, Japan, Sept. 28th - Oct. 2nd, 2004.

[11]Will Leckie and Michael Greenspan, “Monte Carlo Methods in Pool Strategy Game Trees,” 5th International Conference on Computers and Games (CG’06), May, 31, 2006, Turin, Italy.

[12]J. T. Li, J. S. Yang and C. Y. Yang, “Offensive Strategy of a Billiard Robot,” The Eleventh International Symposium on Artificial Life and Robotics (AROB 11th '06), January 23-25, Oita, Japan, 2006.

[13]Deng Julong, Grey System Theory and Application, Full Book Co., Ltd., Republic of 88 years.

[14]Wu Hanxiong Deng Julong, Wenkun Li, grey analysis entry, GAO Books Ltd., 1998.

[15] Wukun Li, Zhao Zhongxian, Erection, grey theory, Hill Book Company, 1998. 